\title{
Carnets
}

Revue électronique d'études françaises de l'APEF

Deuxième série - $18 \mid 2020$

Chiens et écritures (littéraires, filmiques, photographiques)

\section{Cave canem (ou comment un chien méchant peut en cacher un autre)}

\section{Tanguy Wuillème}

\section{(2) OpenEdition}

\section{Journals}

Édition électronique

URL : http://journals.openedition.org/carnets/10885

DOI : $10.4000 /$ carnets. 10885

ISSN : 1646-7698

Éditeur

APEF

Référence électronique

Tanguy Wuillème, "Cave canem (ou comment un chien méchant peut en cacher un autre) », Carnets

[En ligne], Deuxième série - 18 | 2020, mis en ligne le 31 janvier 2020, consulté le 02 juin 2020. URL http://journals.openedition.org/carnets/10885; DOI : https://doi.org/10.4000/carnets.10885

Ce document a été généré automatiquement le 2 juin 2020.

\section{(c) (i) \&}

Carnets est mis à disposition selon les termes de la licence Creative Commons - Atribution - Pas d'utilisation commerciale 4.0 International. 


\title{
Cave canem (ou comment un chien méchant peut en cacher un autre)
}

\author{
Tanguy Wuillème
}

Cave Canem.

Caute.

Méfie-toi.

Prends garde au chien qui menace partout. Prends garde deux fois, c'est-à-dire regarde, c'est-àdire : aie peur» (Quignard, 2012 : 206).

1 Ce fragment-avertissement de Pascal Quignard dans Les désarçonnés sera notre point de départ. Le chien constitue une menace. L'inscription Cave Canem (attention au chien) n'est pas ici qu'un signal linguistique gentillet, l'auteur l'investit d'un sens performatif injonctif. Et il est dérangeant de le rappeler au moment où de nombreuses approches scientifiques, philosophiques ou littéraires, insistent bien davantage sur les bienfaits apportés par Canis Familiaris (chien familier) que sur la peur qu'il devrait engendrer.

Certes, on sait depuis longtemps que ce chien serait un loup qui se serait rapproché de l'homme. Il nous aurait protégés de ses congénères, comme de tout autre prédateur et cela jusqu'aux bons services dont nous lui sommes redevables dans la vie quotidienne. Des origines préhistoriques jusqu'à aujourd'hui, le chien serait devenu notre meilleur compagnon.

3 Pourtant ce fragment introduit un soupçon : attention à ce chien-là, il peut en cacher un autre. Le chien menace partout, la chiennerie est en tout, peut-être même en l'homme. Que faut-il entendre par là, quel avertissement prendrons-nous pour nous, ou pour le dire autrement, quel miroir de notre condition, la figure du chien méchant estelle susceptible nous renvoyer? Nous suivrons à sa suite trois pistes dialectiques et mimétiques :

- Celle première dans le processus de domestication où le chien est élevé, dressé pour obéir. Il se soumet à une servitude volontaire qui dénature sa part animale. Le chien peut se faire méchant sur les ordres de l'homme. Mais l'homme également, dans ses premiers âges, peutêtre gâté à tous les sens du terme, sous l'emprise d'une autorité parentale, familiale, 
magistrale etc. Il devient obéissant envers le social, signe d'une grande grégarité et, par moments, se détermine en tant que méchant. Un commun destin de soumission, puis d'animosité foncière, les rapproche.

- Si l'ontogenèse canine est celle d'un dressage, d'une domestication croissante, il en va tout autrement du travail de la phylogenèse en lui. Il se pourrait que sa véritable nature soit contrecarrée, organisée en fonction d'une obéissance dont il aura beaucoup de mal à se débarrasser, constituant une sorte de seconde nature mais avec l'idée qu'autre chose joue en lui. Même chose en l'homme, une nature primordiale affleure, une phylogénèse œuvre sans fin au fond de son corps : révélé par ses instincts (apports du néodarwinisme), ses pulsions (ceux du Freudisme), par une origine ancestrale encore agissante et déterminante, le chien comme l'homme semblent des êtres voués au désir, désir qui est fondamentalement désir de liberté. Enfin il semble que, si le chien nous montre les inconvénients de l'obéissance, puis les attraits de la liberté, il ne soit pas en mesure, lui, contrairement à l'homme, de réaliser le vrai programme de l'émancipation mais qu'il puisse nous donner des conseils : regarde, aboie, proteste, imite ceux que je chasse méchamment et accepte ton "versant animal » (Bailly, 2018).

4 Rejoins la meute ou bien singularise-toi. Dans l'histoire, cela a pu donner la frugalité cynique, le versant-chien, ou la simple idée de regarder le monde à partir d'un point de vue bassement terrestre. Le chien parvient à définir un autre programme dont il laisse voir en filigrane les potentialités : une politique chienne, afin que l'homme, comme lui, devienne l'objecteur de sa propre histoire, voire même de sa condition. L'homme, tout comme le chien, peuvent vouloir devenir Dieu, Surhomme, robot, cyborg, hybride en tous genres. Ils pourraient se faire crocs et griffes, aidés en cela d'une ironie mihumaine, mi-canine mais également armés d'un certain regard. Et cela dans une méchanceté qui vise une vengeance définitive.

\section{Trois voies pour pister le chien méchant}

5 Une nouvelle particulièrement éprouvante nous permet de poursuivre en détails ces trois pistes du cheminement-chien susceptible de nous servir d'avertissement et d'enseignement politique. Alejo Carpentier dans Les fugitifs (dont l'écriture remonte à 1946), texte qui se trouve dans le recueil Guerre du temps et autres nouvelles (Carpentier, 1967), nous conte une histoire que l'on va résumer brièvement.

6 Il s'agit du marronnage d'un esclave noir qui vient de s'échapper d'une ferme-usine et qui rencontre un chien pourtant lancé à sa poursuite. Chien (il s'appelle ainsi) ne le signale pas et accepte même de quitter la meute pour fuir avec lui. Un autre camp le sollicite. Ils vont faire un bon bout de chemin ensemble dans la forêt, tous deux fugitifs, se réensauvager afin de se nourrir et de survivre. Mais Nègre marron (ce sont les mots employés par A. Carpentier pour le désigner) sera repris. Le chien qui avait quitté un maître blanc pour un autre, noir, va continuer son chemin seul jusqu'à ce qu'il rencontre à son tour une meute de chiens errants avec lequel il va se battre et s'affirmer comme dominant parmi eux. Mais voilà qu'un jour la meute, dont il est le chef, retombe sur Nègre marron qui vient une nouvelle fois de s'échapper :

Chien reconnut Nègre marron:

Chien ! appela le nègre tout joyeux. Chien!

Chien s'approcha de lui lentement. Il flaira ses pieds sans se laisser toucher. Il

tournait autour de lui, en remuant la queue. Quand l'esclave l'appelait, il s'enfuyait.

Quand il ne l'appelait pas, il semblait rechercher le son de sa voix humaine qu'il 
avait compris un peu autrefois, mais qu'il trouvait à présent si étrange, qui évoquait sa vie si pleine de dangers, à la merci du maître. Finalement, Nègre marron fit un pas, avançant vers la tête de l'animal une main prête à caresser. Chien lança un cri étrange, mélange d'aboiement sourd et de hurlement, et sauta à la gorge du nègre.

Il s'était souvenu tout d'un coup, d'une vieille consigne donnée par le commandeur de l'usine, le jour où un esclave prenait le maquis. (Carpentier, 1977 : 148)

\section{Quitter fers et colliers}

7 Cette nouvelle propose plusieurs manières de comprendre le devenir méchant d'un chien. Il a été domestiqué pour la chasse (ici de chasse à l'homme), il a été dressé comme l'esclave lui-même a pu l'être par le maître. On sait depuis l'Antiquité, notamment depuis Xénophon qui en a défini les principaux rudiments dans son traité De la chasse, tout le soin long et coûteux qu'il faut prendre pour obtenir un chien adapté à l'art cynégétique.

8 Tout comme le nègre "bossale ", fraîchement débarqué d'Afrique, indompté, il faut lui mettre une muselière, un mord, une bride et empêcher qu'il retourne à un état sauvage ou qu'il redevienne comme l'esclave un homme-loup, un werwolf, un loup-garou. Leurs méchancetés, à eux deux, doivent rester sous le contrôle du maître. Il s'agit d'un affrontement entre deux méchancetés, construites par le maître, issues par conséquent d'un «pouvoir» animalier contre la possibilité d'un «devenir animal» (au sens de Gilles Deleuze). Le chien de chasse, excité, acharné doit bien rester un gentil toutou de son maître blanc.

9 Les destins croisés de Chien et de Nègre marron nous renvoient ici à la condition obéissante de l'homme, à son élevage, à son dressage afin d'être au service d'un maître (qu'il soit de ferme, de famille, d'école, de stage, de conférences, d'usine...).

10 Certes, on ne peut réduire l'éducation du genre humain à une domestication mais nous sommes forcés de reconnaitre qu'une part de dressage, du corps, des humeurs, de l'attention existe dans le projet pédagogique (il suffit de lire les réflexions pédagogiques de Kant à Sloterdijk, en passant par Deleuze ou Foucault).

11 Le chien garde la maison, le troupeau, l'enfant. Il protège des loups, des prédateurs. Cela évoque ici tous les chiens de traîneaux issus des ouvrages de Jack London, ou encore le Chien blanc de Romain Gary, trouvé dans son jardin, affectueux, mais qui ne cesse d'aboyer après les personnes de couleur noire car il a été élevé à cet effet : « il y a quelque chose de profondément démoralisant, troublant dans ces brusques transformations d'une bête paisible que vous croyez connaître en une créature féroce et comme entièrement autre " (Gary, 1970:14). Les bêtes vicieuses sont des bêtes viciées, délibérément déformées par des années de dressage, avariées. Voilà encore la scène à laquelle assiste, à la fin des années 1960, le narrateur habitant une banlieue cossue en Californie à l'approche d'un dresseur de couleur noire :

Il était à une dizaine de mètres de nous lorsque Batka dressa les oreilles, se figea un instant, puis se leva d'un bond et se jeta en hurlant contre le grillage. Je reçus des gouttes de bave dans la figure. En dehors même de l'image instantanément matérialisée des esclaves en fuite et de ces champs de cotons avec leurs semailles dont l'Amérique n'a pas fini de faire la tragique moisson, il y avait aussi, une fois de plus, ce bouleversement soudain du familier, cette transformation instantanée d'une nature amicale en hostilité sauvage... (Gary, $1970: 21$ ) 

l'ouvrage de Romain Gary qu'il va être à son tour rééduqué et dressé à l'inverse pour agresser les Blancs. Tout le livre décrit la difficulté de la rééducation contre laquelle lutte le chien "d'attaque », avec derrière lui plusieurs lignées spécialement dressées à cet effet, un atavisme lui donnant une seconde nature. Il s'agit bien d'extirper la haine qui a gâté le chien, hélas pour en injecter une nouvelle. éducation qui transpire dans l'ouvrage de Romain Gary : la place des noirs, la haine qui s'abat sur eux, les discriminations, leurs volontés de la renvoyer sur leurs adversaires, sur fond de guerre du Vietnam et d'émeutes raciales. Romain Gary nomme « société de provocation " celle qui est composée d'individus qui obéissent à des mots d'ordre, des idéologies, à des maîtres, aux propagandes et aux publicités environnantes.

Dans un film dont le titre est une référence anagrammatique au livre de R. Gary, Kornél Mundruczo montre dans White God (2014) toute la méchanceté et la cruauté des hommes : dans le scénario, un décret oblige à abandonner des chiens impurs de race à la lisière des villes sous peine d'amende impossible à payer par les familles modestes. Une jeune fille verra avec chagrin partir son chien. La ségrégation joue à plein pour construire un espace civique de l'entre-soi, là où le chien, ailleurs l'homme de couleur, ne saurait pénétrer. Si l'on chasse le chien à la périphérie, il peut également servir à repousser d'autres intrus dans les secteurs protégés. Chien-mirador, chien-barbelé, il est le gardien des espaces totalitaires tout comme doit l'être dans un futur proche le robot-limier du Fahrenheit 451 de Ray Bradbury ou celui entrevu dans un épisode de la série Black Mirror intitulé Metalhead, chien limier hautement perfectionné qui se retourne définitivement contre les humains.

\section{Retournements de situation}

Et précisément le chien peut échapper à toute maîtrise, sa force instinctuelle, intuitive peut reprendre le dessus, se retourner. Cela prend l'aspect de la fuite, ou plutôt de la fugue, de la sécession furtive, du recours aux forêts, de la possibilité d'échapper à la première meute, au conformisme, de quitter les attachements primaires pour acquérir la liberté. Échapper au pouvoir animalier pour un devenir-animal réel. Ne pas être traité comme un chien, devenir un vrai chien, un voisin du loup qui se serait rapproché jadis de l'homme. Il s'agit de retrouver une sorte d'état de nature où le droit des maîtres et le statut de victimes ne s'appliquent plus. coursent, à la curée, tout comme le héros de Bradbury, Montag, échappe au chien robot dans Fahrenheit 451. Notons qu'au xxì̀me siècle, il n'est plus possible d'échapper aux chiens électroniques de Black Mirror, la perfection technique n'étant plus à la mesure des pauvres défenses humaines (le chien en chair et en os a laissé la place à des chiens de plus en plus sophistiqués, obéissant aux ordres mais surtout aux normes et aux diktats d'une technique de prospection infaillible, ceux-là seront précisément difficiles à retourner).

17 Tout retournement suppose dans un premier temps deux options : soit de rejoindre un autre maitre, soit dans un second temps une horde, une meute, une multitude indocile et imperceptible.

Carnets, Deuxième série - 18 | 2020 
On le sait à la lecture de ce chapitre, étonnamment autobiographique de Levinas intitulé « Nom d'un chien ou le droit naturel ». Levinas y salue la présence de ce chien errant dans le camp de concentration, Tobby, qui semble le seul à reconnaître chaque jour les prisonniers, ce pourquoi, nous dit l'auteur, il incarne le «dernier kantien de l'Allemagne nazie » (Levinas, 1963 : 216). doivent se dédomestiquer, s'affranchir. Il faut faire fuir le réel, y opérer des variations pour déjouer toute mainmise. Il s'opère, dans ce texte de Carpentier, une réversibilité des rôles, les voilà devenus eux-mêmes les prédateurs, les chasseurs. Dans un autre ouvrage célèbre de Jack London, Croc-blanc aussi sera libéré par ses derniers maîtres pour rejoindre son territoire de liberté. Tous ces chiens et esclaves marrons suivent une ligne d'ensauvagement, refusant l'assujettissement des esprits et des corps, retrouvant par-là l'esprit américain présenté par David Thoreau ou Mark Twain. pour Chien comme pour Nègre marron de gérer un ensemble de violences pour survivre dans la jungle et ne pas être repris par le Maître d'usine. Ce dernier étend encore son ombre menaçante mais en bas dans la forêt, une nouvelle égalité se dessine dans le couple affranchi. Le chien opère la médiation entre les hommes et incarne la circulation des désirs de liberté. Ce Chien est tiraillé entre deux possibilités : opter pour une vraie liberté sans aucun maître ou choisir l'accompagnement fidèle d'un nouveau 
maître, même relatif, afin d'apprendre les recettes de la survie. La dialectique hégélienne du maitre et de l'esclave se dote d'un tiers, le Chien : il est deux fois esclave (du premier maître absolu de l'usine, puis semi-esclave libre aux côtés du Nègre marron) et voilà qu'il va se mettre à occuper une place entièrement libre éloignée des deux maitres, en devenant le guide d'une nouvelle meute de chiens ainsi que celui de la forêt. Il ne deviendra maître que pour mener la multitude de ses égaux (même si sa force le distingue d'entre tous), en vue de retrouver son écosystème sauvage. On verra que cette multitude se compose non pas de collectifs concurrentiels, mais de singularités actives autour d'un foyer commun.

Dans ce second stade, si le chien copie l'homme, l'homme en fait de même aux côtés du chien. Cette part sauvage, c'est la métamorphose progressive de l'homme en animal: l'affût chasseur, la faim qui tenaille, la ruse. L'animalité précède l'humain, qui lui permet de devenir terrestre. La condition animale demeure immanente et chasse toute autre transcendance. La conquête de la liberté suppose de retourner à un autre état de violence. Après la violence de la domination de l'homme sur l'homme, éprouvée dans le monde politique du travail esclave, après celle de l'homme sur le chien du fait de la férocité du maître, les voilà tous deux, à même hauteur de sol, qui violentent le réel pour survivre. Le lien originaire à la nature et à la vie primitive a remplacé celui de la société dite civile faite d'exploitation, de commandement et d'obéissance.

\section{Sortir de la nuit animale : Dog Liberation}

Cependant, comme l'indique la nouvelle de Carpentier, la fugue va tourner court pour le Nègre marron alors que le chien, lui, va devoir s'affirmer dans une meute de congénères errants.

Et lorsqu'il tombe de nouveau face à son ancien acolyte, il ne le reconnaît pas. Ou du moins pas comme le voudrait le modèle mythique : on se souvient d'Argos qui peut enfin mourir, vingt ans après le départ d'Ulysse. Il était dans l'odyssée le seul chien digne de son maître, le seul à avoir un nom car il est le prolongement d'Ulysse, son double qui garde la maison en son absence. Dans la nouvelle de Carpentier, Chien va dévorer Nègre marron car « il s'était souvenu tout à coup d'une vieille consigne donnée par le commandeur de l'usine ». On est plus proche d'un autre mythe narré par Ovide dans le livre III des Métamorphoses, celui d'Actéon et de ses nombreux chiens qui finiront par le dévorer.

27 Ainsi se présentent trois premières variantes explicatives possibles de ces métamorphoses de Chien: soit il présente toutes les caractéristiques de l'homme vicieux car il a été mal élevé. Soit il acquiert la sophistication même de la réponse automatique et robotique issue aussi de l'ingéniosité humaine mais augmentée technologiquement tel qu'on peut le voir dans l'épisode Metalhead de la série Black Mirror. Soit le Chien devient un Dieu, le White God, rappelant les anciens dieux cynocéphales de l'Antiquité ou encore le porteur du Christ, Saint Christophe souvent représenté avec une tête de chien.Le chien-dieu devient le conducteur de la communauté, celui qui sauve du péril humain.

Il faut reprendre ces trois premières possibilités.

Soit la dédomestication ne s'est pas faite à plein. Il y a un reste de violence, d'ordre donné par une ancienne maîtrise chasseresse comme dans l'exemple de la nouvelle de 
Carpentier. On passe là encore d'une métamorphose à une autre : l'animal dressé pour la chasse à l'homme devient libre, mais font alors retour les anciennes consignes du dressage.

Soit on peut aussi voir cela avec un autre œil : l'arme de l'homme se retourne contre lui, tout ce qui est homme mérite la mort. Le chien, sophistiqué par la main violente, s'affirme outre-mesure, même contre son maître, il est devenu le maître survivant et implacable. Il en va ainsi également dans l'épisode de la série Black Mirror (Metalhead), le limier-robot-chien est imparable et nul ne peut lui échapper, l'engin technologique s'est perfectionné avec l'ingéniosité humaine. Le chien a su se faire homme, quand l'homme n'a pas su apprendre du chien.

En revanche, la métamorphose pour l'homme n'a pas joué à plein, ou du moins, comme dans le mythe d'Actéon, l'homme a choisi le mauvais animal : il se transforme en cerf, animal pourtant roi de la forêt, hautement solitaire et singulier, mais qui ne peut rien contre la meute de chiens. Un homme doit se métamorphoser en chien-homme (cynocéphale ou cyno corporel) pour survivre, sinon il meurt dans la déchirure des crocs.

31 Soit troisième possibilité, le chien gagne le sommet de la pyramide vivante : comme l'exemplifie le film de Kornél Mundruczo, White God (2014): le Dieu ne l'est plus, remplacé par Dog devenu God. Mark Alizart (2018) montre combien le monothéisme avait fait de la figure divine et de ses représentants incarnés, les seuls gardiens de seuils et des passages, rôles autrefois attribués aux chiens dans les croyances grecques ou romaines. Le Christ (puis saint Christophe) a pris la place du chien. Ensuite l'homme nietzschéen a occupé la place de Dieu. Ici on pourrait compléter cette suite métamorphique, le Chien prend la place de l'homme et celle de Dieu. Trois lettres, D-O$G$, peuvent en cacher d'autres. Dans le film de Mundruczo, le chien-dieu vengeur sort de la banlieue où il a été relégué pour revenir sur les lieux des exactions et des meurtres humains: accompagné de tous les bâtards qu'il a réunis, il attaque les abattoirs, les fourrières, les chenils, les boucheries, les cabinets de vétérinaires, les zoos...Ce n'est plus le scénario anticipateur de la planète des singes mais celui du règne animal, d'abord canin, qui demande réparation au genre humain et vient proposer plusieurs nouvelles configurations de coexistence.

\section{Après la nuit animale, un devenir-chien de l'homme?}

On l'a vu dans la dernière des trois variantes, Chien qui a pris le poste de chef de meute propose une solution payante : comme dans White God, le film de Mundruczo (2014), le chien abandonné revient pour terroriser la ville humaine qui l'a rejeté (sauf l'enfant qu'il finit par reconnaître). On est face aux prémices de l'apocatastase, d'un passé animal qui est restitué dans son intégralité, de la vengeance des animaux revenus à la vie qui s'affirment pour contester la suprématie humaine.

33 Cependant si l'animal est devenu chef de meute, s'il a fait multitude, d'autres fictions que celle de Carpentier ou de Mundruczo nous présentent encore des alternatives politiques. Alternatives supplémentaires qui ne sont pas seulement agrégatives ou collectives, mais qui font signe vers des options davantage singulières.

Là encore trois possibilités marquées toute par une errance qui préserve la liberté. Ce peut être celle présentée par le Croc-blanc de Jack London: le devenir singulier de 
l'animal et surtout du chien solitaire qui rejoint sa vraie nature, ou celui de Buck vu plus haut, qui trouve une famille et va gagner son territoire, son cri, sa voix.

Une seconde possibilité se dessine : redevenir un de ces chiens errant à la frontière des villes, à la lisière de la civilisation comme pour de nouveau garder les seuils, les passages vers d'autres mondes. Ce sont des chiens qui s'effacent, disparaissent pour mieux résister, chiens qui savent se suffire de peu contre l'obésité dictatoriale de l'homme.

Il est une phrase étrange de Walter Benjamin que l'on peut commencer à comprendre : « tant qu'il y aura encore un mendiant, il y aura encore du mythe » (Benjamin, 1989: 417). Ce peut être ce chien qui fait les poubelles, qui se nourrit des restes, comme le chiffonnier d'autrefois ou comme Diogène qui mendie une obole à une statue pour s'exercer à ne rien recevoir, pas même un morceau de sucre. Il s'agit d'adopter une attitude hautement «cynique», celle qui sait supporter la pauvreté pour parvenir à une nouvelle liberté et à une réelle franchise dans l'existence.

Où l'on retrouve l'échange entre Alexandre le Grand et Diogène. "Qui es-tu? » Je suis Alexandre le Grand! Je suis Diogène le chien. Ce dernier s'affirme dans une extériorité absolue de personne philosophique, libre à l'encontre du personnage royal. Ce que soutient Diogène Laërce qui en fait le récit. Il est chien car il caresse ceux qui donnent, il aboie contre ceux qui ne donnent pas et mord ceux qui sont méchants. Il s'agit d'accepter le pouvoir mais de rester libre à son égard et parfois violent pour dire ce qu'on veut, affirmer ce qui est vrai et ce qui est juste.

Ce chien qui se distingue donne à voir une singularité, dans l'écart, une déprise sociale et solitaire. Il peut se déterminer face à la super-meute sociale. L'attitude qu'il nous propose consiste à perdre toute identité, à se rendre anonyme, ou du moins à se désidentifier afin paradoxalement de retrouver une sorte de "vrai soi» au sens de Winnicott. Ce moi asocial, a-linguistique, réaffirme en l'homme sa part animale. Et cela passe par l'adoption pour chacun d'un nouveau point de vue animal sur soi-même.

Ce peut être également, non pas faire de nouveau meute, ni de rester totalement esseulé et inconsolable comme Hachiko le chien fidèle à son maître décédé dans le film de Tatsuya Nakadai (1987), qui chaque soir vient l'attendre devant la gare. Mais ce serait rejoindre une communauté de solitaires. Ainsi la multitude et les singularités se liguent pour une entreprise politique critique et radicale.

Cette figure se retrouve également dans la dernière figure, celle du chien errant qui accompagne Charlot. Il est son alter ego et chacun s'apporte un soin réciproque. Tous deux font de leur pauvreté un effort, un moyen de connaissance et abandonnent le gémissement canin de derrière la porte. Le chien montre ici combien il faut se délester des leçons apprises du système ou de la machinerie. Il faut oublier et se réveiller de ce que fut l'existence du passé de nos entourages. Le chien appauvri trouve des ressources en se débrouillant, il quitte le monde humain pour errer et déterrer des portefeuilles. Il retrouve les qualités de l'enfance, le plaisir de jouer, d'avoir un corps, de parler à la nature, de sentir comme un chien à la manière de Botte et Pantoufle, les chiens de Kipling (2010). Cela passe par un style de vie où se mêlent l'improvisation, une curiosité tous azimuts, une perméabilité au monde, de la gaieté, un rêve de liberté. Le clochard peut aussi se trouver une belle. 
41 Les chiens de Chaplin rêvent d'un monde meilleur, d'une autre vie, ce sont des chiens utopiques quand l'homme ne sait plus penser à autre chose qu'à la défense de ses intérêts.

\section{L'appel de la métamorphose}

Le chien nous indique bien plusieurs possibilités de mener sa vie en vue de plus d'autonomie et d'authenticité, les deux projets de l'homme contemporain. Les circonstances appellent à des transformations : il peut s'agir de lutter en retrouvant des vestiges cruels de notre part animale, on peut se perfectionner et se doter de prothèses post-humanistes, imparables pour l'ennemi, on peut encore séduire et guider une meute d'affamés et religieusement combattre les oppresseurs. Puis pour finir il y a ces errances positives, où le chien retrouve sa voix, garde les seuils et joue avec le monde.

Toutefois il y a encore de la place pour d'autres métamorphoses concernant l'homme au regard du chien, ce que dit autrement Pascal Quignard dans un paragraphe consacré à Ovide : «l'anthropomorphose n'est pas achevée. (...) Ovide : L'homme doit être laissé comme non fini, c'est-à-dire comme appartenant à une espèce en cours de métamorphose infinie dans une nature qui est elle-même une métamorphose infinie " (Quignard, 2012 : 131).

On assiste bien avec ces récits et ces films à des déplacements, au brouillage des frontières des points de partage entre humanité et animalité. Les narrateurs montrent la profondeur d'un devenir-chien contre la bêtise humaine (contre "la connerie humaine " selon les mots de R. Gary, les Blancs comme les Noirs qui pourrissent les chiens, qui tournent et retournent le chien ainsi devenu un Black Dog), contre ces dresseurs qui, selon lui, mériteraient d'être piqués, comme d'autres le pratiquent sur les chiens. Sans être si extrême, le chien ange ou démon est révélateur d'un trouble dont il faut s'approcher.

Le chien errant, clochard futé et débrouillard est aussi celui qui permet de contester l'histoire, d'intégrer celle-ci au sein d'un plus vaste récit, qu'il soit celui de la liberté animale, ou celui de l'origine propre de l'homme. Ce chien ici entrevu apparait déjà dans certains tableaux anciens, en une avant-scène qui semble se moquer des expériences humaines: chiens qui se tiennent dans l'ombre de cette estampe de Domenico Campagnola, Bataille d'hommes nus (1517 Louvre) et qui semble aboyer contre la guerre des hommes, protester contre leur cruauté. Dans cette planche dessinée, les hommes ne sont plus armés ou casqués mais tous nus et là, petit, au bas de la planche, le chien aboie, il est l'objecteur de leur hubris ou encore comme le dit Georges DidiHuberman (2018) à propos du chien de La ronde de nuit de Rembrandt, l'objecteur de leur Histoire.

Là encore, nous serions en présence du chien glapisseur, aboyeur qui peste contre le désordre du monde. C'est aussi un chien qui voit quelque chose, qui pose son regard sur la violence humaine. Un jour futur, il prendra davantage son temps pour nous ausculter, tout en vivant avec nous comme le Niki de Tibor Déry (2010), tout en nous invitant à nous mettre à sa hauteur, à la mesure de son regard, de son flair et de sa réflexivité. Il se peut que Romain Gary ait bien vu cette nouvelle perspective à gagner quand il nous dit que «le seul endroit au monde où l'on peut rencontrer un homme digne de ce nom, c'est le regard d'un chien » (Gary, 1970 : 154). 
Le chien ne sait pas encore s'organiser comme la classe ouvrière, ni même revendiquer ses droits sans l'aide de l'homme, il ne peut comme l'oiseau échapper au pouvoir de l'homme mais il sait déjà errer auprès des seigneurs, près de leurs lieux de violence et signifier la libération animale en marche. Il parvient encore à reconnaitre la part de bonté de certains humains mais sa vengeance contrôlée pourrait en cacher une autre.

\section{BIBLIOGRAPHIE}

ALIZART, Mark (2018). Chiens. Paris : PUF.

BAILLY, Jean-Christophe (2018). Le versant animal. Paris : Bayard.

BENJAMIN, Walter (1989). Paris capitale du XIX siècle. Paris : Cerf.

CARPENTIER, Alejo (1977). Guerre du temps et autres nouvelles. Paris: Gallimard.

DÉRY, Tibor (2010). Niki. Belval: Circé.

DIDI-HUBERMAN, Georges (2018). Aperçues. Paris : Minuit.

GARY, Romain (2010). Chien blanc. Paris : Folio Gallimard.

KIPLING, Rudyard (2010). Paroles de chien. Paris : Rivages.

LEVINAS, Emmanuel (1963). Difficile liberté : essais sur le judaïsme. Paris : Albin Michel.

LONDON, Jack (2000). L'Appel de la forêt. Paris : Le livre de poche.

PALUMBO, Jonathan (2018). Après la nuit animale. Paris : Marest.

QUIGNARD, Pascal (2012). Les désarçonnés. Paris: Folio Gallimard.

\section{Filmographie}

MUNDRUCZO, Kornél (2014). White God.

NAKADAI, Tatsuya (1987). Hachiko.

Série Black Mirror, épisode Metalhead (2017), Netflix.

\section{RÉSUMÉS}

Loin des approches convenues des vertus et qualités du meilleur compagnon de l'homme, cet article décline les devenirs méchants du chien. En partant d'une nouvelle d'Alejo Carpentier, il montre combien le chien peut subir de transformations: dressé pour la chasse aux animaux comme à l'homme, il peut vouloir retrouver sa liberté en s'affirmant violent. Il peut également ne plus vouloir reconnaître la suprématie humaine et faire signe vers une émancipation politique radicale par le biais de la meute ou d'une communauté autrement plus singulière. En mobilisant tout un ensemble de récits et de films, on perçoit comment le chien peut se métamorphoser, indiquant à l'homme les ressources pour sa propre autonomie. En vue d'empêcher une vengeance destructrice des animaux envers leurs maîtres, une apocatastase animale, la figure du chien 
méchant pourrait constituer un modèle de vie émancipée de toute tutelle et combinant les destins croisés et créatifs du chien et de l'homme.

Far from the standard approaches of the virtues and qualities of the Man's Best Friend, this article explores the evil futures of the dog. Starting from a short story by Alejo Carpentier, we show how many transformations the dog can suffer: trained for hunting beasts as well as men, he can wish to regain his freedom by asserting his violence. He can also cease to acknowledge human supremacy in a sign of radical political empowerment through the pack or a rather more unusual community. Through a set of stories and movies, we discern how the dog can morph, showing to the men the resources of their own autonomy. In order to prevent a destructive revenge of the animals against their masters, an animal apocatastasis, the figure of the bad dog could serve as a model of a life freed from any supervision, combining the creative destinies of the Dog and the Man.

INDEX

Keywords : bad Dog, metamorphosis, Carpentier (Alejo), politics, dog liberation

Mots-clés : chien méchant, métamorphose, Carpentier (Alejo), politique, libération des chiens

\section{AUTEUR}

\section{TANGUY WUILLÈME}

Université de Lorraine

tanguy.wuilleme[at]univ-lorraine.fr 